mehr Episoden erlebt. Ein signifikanter Zusammenhang zwischen Schlafdauer und Selbstverletzung konnte gezeigt werden: So war die durchschnittliche Schlafdauer um etwa eine Stunde kürzer in der Gruppe der Jugendlichen mit selbstverletzendem Verhalten (5,33 vs. $6,29$ Stunden; $p<0,001)$. Signifikante Unterschiede zeigten sich auch bei Einschlafzeit, Erwachen nach Einsetzen des Schlafes und Schlafmangel. Zum Ausmaß der Schlafprobleme wurde festgestellt: $48 \%$ der Jugendlichen mit mehrmaligen selbstverletzenden Episoden litten an Insomnie, während dies nur auf $37 \%$ der Jugendlichen mit nur einer Episode zutraf ( $\mathrm{p}<0,001)$. Depression erklärte einen Teil des Zusammenhangs zwischen Schlafproblemen und Selbstverletzung, aber nicht Perfektionismus und ADHS.

Die Studie zeigt einen starken Zusammenhang zwischen Schlafproblemen und selbstverletzendem Verhalten. Interventionen für betroffene Jugendliche sollten Schlafprobleme als Therapieziel mit einschließen.
Hysing $M$ et al. Sleep problems and self-harm in adolescence. Br J Psychiatry 2015;207:306-12

\section{Kommentar}

Die populationsbasierte Querschnittstudie (Evidenzniveau III - IV) konnte den bereits zuvor in der Literatur beschriebenen Zusammenhang zwischen Schlafproblemen und selbstverletzendem Verhalten bei Jugendlichen bestätigen. Die Autoren konnten aufzeigen, dass dieser Zusammenhang trotz Berücksichtigung von Depression und weiterer Co-Variablen signifikant bleibt. Es handelt sich hier jedoch um die erste Studie, die in diesem Zusammenhang eine Vielzahl von Parametern bezüglich des Schlafes untersuchte und somit eine differenzierte Betrachtung ermöglicht. Es ist jedoch zu beachten, dass die Parameter allesamt mittels Fragebogen erhoben wurden und somit auf Selbsteinschätzung der Befragten beruhen, was ein gewisses Risiko für systematische Fehler bedingt. Zudem können aufgrund des Studiendesigns keine Aussagen zu Kausalität oder zum Zeitverlauf getroffen werden. Folgerichtig betonen die Autoren die
Notwendigkeit der Durchführung von Längsschnittstudien. Die in der vorliegenden Studie geschätzte Prävalenz von selbstverletzendem Verhalten liegt mit $7,2 \%$ unterhalb dessen, was vorhergehende Arbeiten festgestellt hatten [Hawton $\mathrm{K}$ et al. Lancet 2012;379:2373-82]. Aufgrund des Umstands, dass Nichtteilnehmer oftmals eine ausgeprägtere psychische Problematik aufweisen [Stormark KM et al. J Abnorm Child Psychol 2008;36:411-9], sollte bei der Frage nach der Generalisierbarkeit der Ergebnisse die Teilnehmerquote von $53 \%$ bedacht und die Möglichkeit der Unterschätzung des Vorkommens von selbstverletzendem Verhalten, Schlafproblemen und depressiver Symptomatik berücksichtigt werden - wie auch die Autoren einräumen. Nichtsdestotrotz bietet die vorliegende Studie wertvolle Anregungen zur Hypothesengenerierung für zukünftige Forschung auf dem Gebiet. Die Berücksichtigung von Schlafproblemen innerhalb von Interventionen bei selbstverletzendem Verhalten erscheint im Rahmen von entsprechenden klinischen Studien sinnvoll.

Dr. Jessica Mattivi

\title{
Laser-Akupunktur ist effektiv beim neonatalen Drogenentzugssyndrom
}

Raith W et al. Laser acupuncture for neonatal abstinence syndrome: a randomized controlled trial. Pediatrics 2015; 136: 876-884

\section{Die Zahl von Neonaten nach intrauteriner Drogenexposition (NAS) nimmt weltweit zu. Eine österreichisch-kanadische Arbeitsgruppe hat es sich nun zur Aufgabe gemacht, den Effekt einer additiven Laser-Akupunktur bei neo- natalem Drogenentzug zu erforschen. Diese Arbeit wurde vor kurzem in Pediatrics publiziert.}

Ü ber einen Zeitraum von gut 5 Jahren zwischen 2009 bis 2014 wurden 28 Neugeborene mit NAS nach maternaler Opioid-Substitutionstherapie während der Schwangerschaft behandelt. Sie wurden zwecks Drogenentzugstherapie in eine Akupunkturgruppe (Kombination von Laser-Akupunktur und pharmakologischer Therapie mit Morphin und Phenobarbital) und Kontrollgruppe (ausschließlich pharmakologische Therapie) eingeteilt.

Die Laser-Akupunktur wurde mit dem LABpen MED 10 (Behounek, Österreich) durchgeführt, einem „continuous wave semiconductor" GaAs-Laser
(Wellenlänge $675 \mathrm{~nm}, 10 \mathrm{~mW}$, Durchmesser 1,5 mm). Die Akupunktur erfolgte täglich an fünf Ohr- und vier Körperpunkten bilateral. Primärer Zielparameter war die Dauer der Morphintherapie. Sekundäre Parameter waren der höchste Finnegan-Score, Gesamtmorphinmenge und weitere zeitliche Parameter.

Im Vergleich zur Kontrollgruppe war sowohl die Dauer der oralen Morphintherapie in der Akupunkturgruppe signifikant kürzer (28 versus 39 Tage, $\mathrm{p}=$ $0,019)$ als auch die Krankenhausaufenthaltsdauer (35 versus 50 Tage, $\mathrm{p}=$ 0,048).

\section{Kommentar}

Eine neonatologische Einrichtung kann sich glücklich schätzen, wenn sie einen erfahrenen Akupunktur-Anwender wie den Erstautor dieser Untersuchung, PD Dr. Wolfgang Raith von der Klinischen Abteilung für Neonatologie in Graz, in ihren Reihen hat Aufgrund der geringen Patientenzahl und der Studienplanung hat diese Untersuchung natürlich nur einen "Pilot-Charakter". Es fehlen bislang auch Langzeitdaten zu Verhalten, neurologischer Entwicklung und Kognition der behandelten Kinder. Grundsätzlich ist eine Lasertherapie jedoch bei entsprechender Expertise des Behandlers sicher, praktikabel, effizient und gut verträglich - gleichermaßen für Patienten wie Angehörige. Wo immer möglich, sollte diese additive Therapieform in die multimodale Therapie des neonatalen Abstinenzsyndroms aufgenommen werden.

Dr. Thomas Hoppen 